\title{
THE EFFECTS OF ACYCLOVIR ON ANGIOGENESIS IN CHICK CHORIOALLANTOIC MEMBRANE MODEL
}

\author{
İbrahim Kılıççalan ๑, Bekir Nihat Doğrul @, Abdulbaki Erkovan ๑), Ekrem Samet Aşçı $\odot$
}

Gülhane School of Medicine, University of Health Sciences, Ankara, TURKEY

\begin{abstract}
Aims: This study aims to reveal the effects of acyclovir on angiogenesis and to assess the experimental doses. Methods: In the study, the chick chorioallantoic membrane model was used as an experimental model. Forty fertilized eggs were kept at $85-90 \%$ relative humidity, below $37^{\circ} \mathrm{C}$ until the fifth day post-fertilization, when the vessels in the chick chorioallantoic membrane model appeared and the drugs were applied. Four different concentrations of acyclovir were chosen to determine the mode of action and dose dependence: $3.55 \mathrm{mg} / \mathrm{mL}, 7.1 \mathrm{mg} / \mathrm{mL}, 14.2 \mathrm{mg} / \mathrm{mL}$, and $28.4 \mathrm{mg} / \mathrm{mL}$. Each of the $1 \mathrm{~mL}$ total acyclovir concentrations were applied to the chick chorioallantoic membrane surfaces. The chick chorioallantoic membranes treated with sterile distilled water were designated as controls. Eight eggs were used for each test group. After applying the drug, all the eggs were covered with transparent tape and kept under the same conditions throughout the experiment. The results were evaluated 48 hours after the drugs were administered and the results were recorded with a digital camera. Results: In our study, it was observed that $3.55 \mathrm{mg} / \mathrm{mL}$ acyclovir concentration shows the reduced density of capillaries around the pellet and no change in the number of capillaries. Both $7.1 \mathrm{mg} / \mathrm{mL}$ and $14.2 \mathrm{mg} / \mathrm{mL}$ concentrations of acyclovir caused a local reaction that was restricted to the membrane and it was attributed to local crystallization reaction. The concentration of $28.4 \mathrm{mg} / \mathrm{mL}$ had a toxic effect on the eggs. Conclusion: In this study, it was found that acyclovir has a very weak anti-angiogenic effect dose-dependently at the concentrations used. Considering that an observational model was used in our study, quantitative studies are needed for assessing anti-angiogenic effects in the future. There is also a need for further studies to elucidate the effects of acyclovir on vascular endothelial growth factor level and which stage of the angiogenesis-related process it is specifically effective on. Keywords: Angiogenesis, acyclovir, chorioallantoic membrane, endothelial cells
\end{abstract}

\section{INTRODUCTION}

Acyclovir (acycloguanosine) is an antiviral drug that inhibits DNA synthesis and viral replication. In doing so, its toxicity to host cells is low, and its concentration in herpes simplex virus (HSV)-infected cells are 40-100 times higher than in normal cells $(1,2)$. Acyclovir is available in oral, topical, and intravenous forms. It is used for HSV-1, HSV-2, and varicella-zoster virus (VZV) infections that can cause herpetic keratitis, herpetic encephalitis, and genital herpes $(3,4)$. Acyclovir is still the gold standard treatment today for HSV infections (5). In addition to treatment, acyclovir prophylaxis also plays an important role in medicine $(6,7)$. However, it has been shown in the literature that acyclovir acts by increasing apoptosis in some cancer lines $(8,9)$. This situation paves the way for studies on the use of acyclovir in different treatment protocols.

Angiogenesis is defined as the formation of new capillaries from existing capillaries. It plays a role in many physiological processes such as wound healing, growth, and development of organs, and takes a part in pathological processes like cancer development. Chick chorioallantoic membrane (CAM) assay is an experimental model that is generally used to study in vivo angiogenesis, tumor cell invasion, and metastasis (10).

As an antiviral drug, acyclovir has been studied in other areas besides HSV and VZV. However, studies investigating its effect on angiogenesis are insufficient. In this study, we aim to examine the effect of acyclovir on angiogenesis using the chick CAM model with four different concentrations.

\section{MATERIAL AND METHODS}

The CAM model was used in our study. CAM model is frequently used in studies due to its low cost, easy use, and real-time visualization of the model (11). In our study, 40 fertilized eggs of chicken were purchased from a poultry institute in Ankara, Turkey. Eggs were kept in an incubator at $37{ }^{\circ} \mathrm{C}$ and $85-90 \%$ relative humidity for five days before drug application. Incubated eggs were confirmed after vascularization appeared on CAM. To open a small window in the egg, the upper part of the eggshell is peeled without damaging the embryonic structures. Acyclovir (Zovirax $250 \mathrm{mg}$; GlaxoSmithKline) for intravenous administration was diluted with distilled water. Four different concentrations of acyclovir were chosen to determine the effectiveness, dose-dependency, and toxicity.

In human pharmacokinetic studies, when acyclovir (Zovirax) was administered at a dose of $5 \mathrm{mg} / \mathrm{kg}$, its Cssmax was $9.8 \mathrm{mcg} / \mathrm{mL}$, its Csstrough was $0.7 \mathrm{mcg} / \mathrm{mL}$; when administered at a dose of $10 \mathrm{mg} / \mathrm{kg}$, its Cssmax was found to be $22.9 \mathrm{mcg} / \mathrm{mL}$, and its Csstrough as $1.9 \mathrm{mcg} / \mathrm{mL}$ according to the United States Food and Drug Administration (12). Doses were determined in the study to cover the therapeutic range in humans. For this purpose, acyclovir was administered to the eggs at concentrations of $3.55 \mathrm{mg} / \mathrm{mL}$, 
$7.1 \mathrm{mg} / \mathrm{mL}, 14.2 \mathrm{mg} / \mathrm{mL}$ and $28.4 \mathrm{mg} / \mathrm{mL}$, respectively. Eight eggs were used for each test group. $50 \mu \mathrm{L}$ aliquots of $1 \mathrm{~mL}$ total acyclovir concentrations were applied to the CAM surfaces. The control group was treated with distilled water only. After applying acyclovir, all the eggs were covered with paraffin film to prevent dehydration and placed back in the incubator. All eggs were kept under the same conditions throughout the experiment.

The results were evaluated 48 hours after the medications were administered, as advised by standard CAM experiment protocols (11). Visualization of blood vessels was recorded with a digital camera (Konica Minolta, Tokyo, Japan). The reduced density of capillaries around the drug application area was considered as an indicator of the anti-angiogenic effect $(13,14)$. A scoring system used in many previous studies was used to evaluate the anti-angiogenic effects (Table 1) $(15,16)$.

\section{RESULTS}

Two days after the application of acyclovir solutions, the vascular network of the treated area of the membrane presented several macroscopic changes compared to the untreated areas of egg membrane, as untreated areas can be observed in Figure 1.

In the area applied at the concentration of $3.55 \mathrm{mg} / \mathrm{mL}$, there was reduced macroscopic density, and no increase in the number of capillaries was observed (Figure 2). Physiological angiogenesis was observed in the areas where acyclovir was not applied. This observation was assessed on a score of 0 to 0.5 which means a very weak anti-angiogenic effect (Table 1). At the concentrations of $7.1 \mathrm{mg} / \mathrm{mL}$ and $14.2 \mathrm{mg} / \mathrm{mL}$, we were unable to interpret any anti-angiogenic or angiogenic effects due to local reaction (Figure 3, Figure 4).

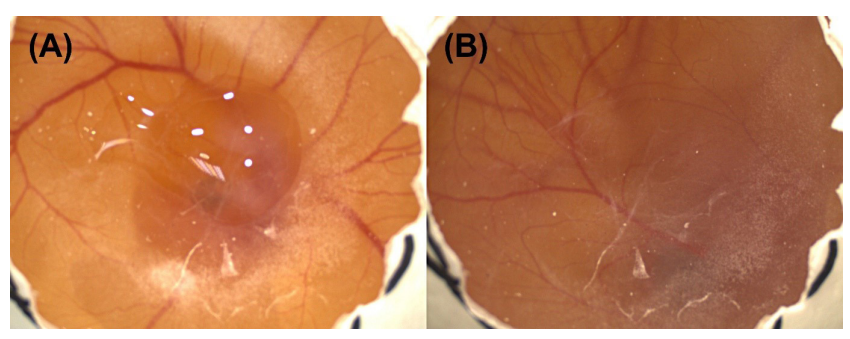

Figure 1: Images of capillary plexus of the CAM treated with sterile distilled water. A: Image recorded at day 0 (five-day-old chick embryo). B: Image recorded after the administration of the sterile distilled water as a control (seven-day-old chick embryo).

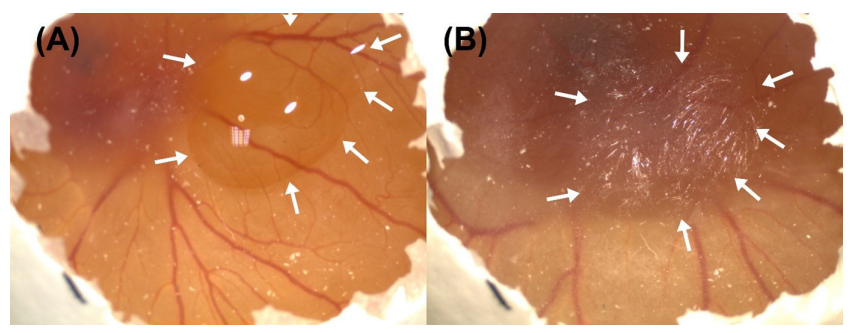

Figure 3: Images of capillary plexus of the CAM treated with 7.1 $\mathrm{mg} / \mathrm{mL}$. A: Image recorded at day 0 (five-day-old chick embryo). (The white arrows show the boundaries of the area affected by the applied substance.) B: Image recorded after the administration of the drug (seven-day-old chick embryo). The local crystallization reaction made observational evaluation difficult (The white arrows show the boundaries of the area affected by the applied substance).
Table 1: Scoring system for the evaluation of the anti-angiogenic effects on chick chorioallantoic membrane model $(15,16)$.

\begin{tabular}{ll}
\hline Score & Effects observed \\
\hline 0: No effect & -None \\
0.5: Very weak effect & -No capillary-free area \\
& -Area with reduced density of capillaries \\
around the pellet not larger than the area \\
of the pellet
\end{tabular}

A toxic effect was detected in the application area with surrounding vascular structure at the concentration of $28.4 \mathrm{mg} / \mathrm{mL}$ (Figure 5). It was concluded that the concentration of $28.4 \mathrm{mg} / \mathrm{mL}$ acyclovir is the toxic dose to eggs because all eight eggs treated with $28.4 \mathrm{mg} / \mathrm{mL}$ showed global toxic effects as the membrane structures were destroyed.

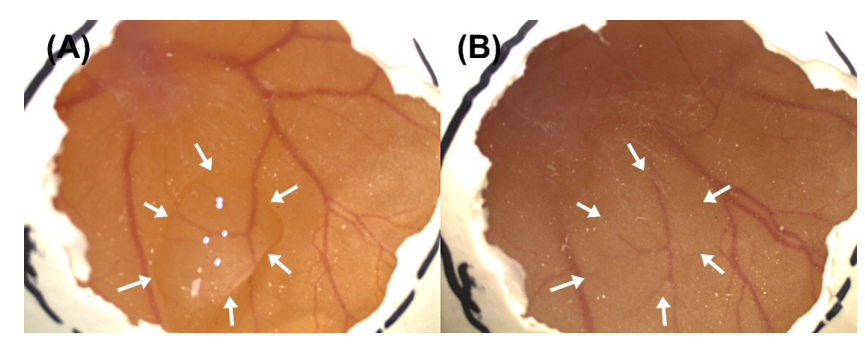

Figure 2: Images of capillary plexus of the CAM treated with 3.55 $\mathrm{mg} / \mathrm{mL}$. A: Image recorded at day 0 (five-day-old chick embryo). (The white arrows show the boundaries of the area affected by the applied substance.) B: Image recorded after the administration of the drug (seven-day-old chick embryo). Minimal to moderate reduced density of capillary can be seen. But the other areas other than pellet shows physiologic angiogenesis with increased density of capillaries and sprouting of vessels (The white arrows show the boundaries of the area affected by the applied substance).

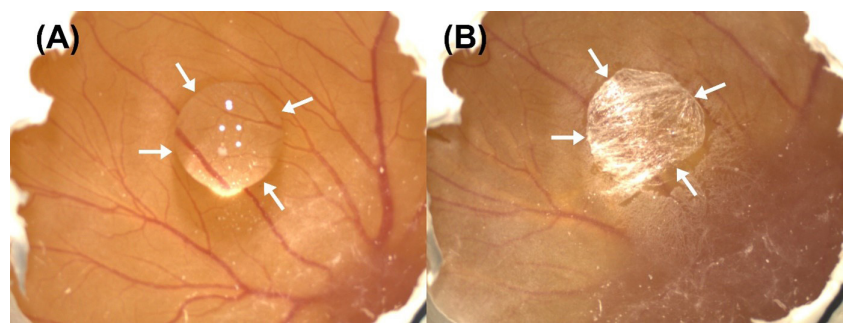

Figure 4: Images of capillary plexus of the CAM treated with 14.2 $\mathrm{mg} / \mathrm{mL}$. A: Image recorded at day 0 (five-day-old chick embryo). (The white arrows show the boundaries of the area affected by the applied substance.) B: Image recorded after the administration of the drug (seven-day-old chick embryo). The local crystallization reaction made observational evaluation difficult (The white arrows show the boundaries of the area affected by the applied substance). 


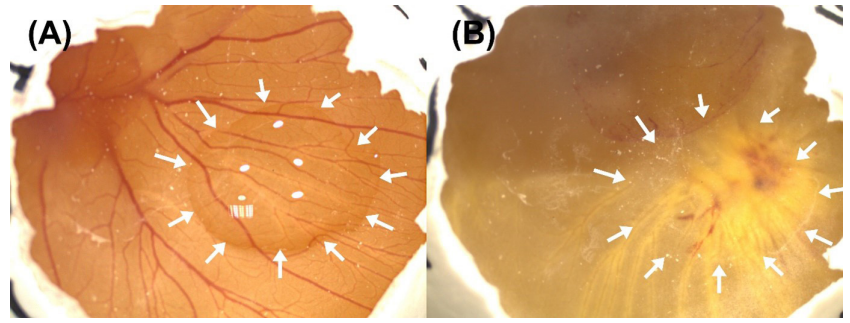

Figure 5: Images of capillary plexus of chick CAM treated with $28.4 \mathrm{mg} / \mathrm{mL}$. A: Image recorded at day 0 (five-day-old chick embryo). (The white arrows show the boundaries of the area affected by the applied substance.) B: Image recorded after the administration of the drug (seven-day-old chick embryo). Global toxicity can be seen (The white arrows show the boundaries of the area affected by the applied substance).

\section{DISCUSSION}

Angiogenesis is the generation of new vessels from existing vessels. It occurs physiologically or pathologically in the body (17). CAM is a simple and in vivo model that is suitable for showing whether a substance is angiogenic or anti-angiogenic. Being able to perform the assay and make an observation by directly accessing the membrane is one of the advantages of the CAM model, but also it is more physiological than in vitro models (18). Besides its simplicity, it is a great advantage of the CAM study that the fertilized egg does not require ethical approval until it reaches 14 or 17 days of development in most countries (19). However, quantification of angiogenesis is usually not easy as it is based on visual observation.

Acyclovir is an antiviral drug used in HSV-1, HSV-2, and VZV infections $(3,4)$. However, publications show that acyclovir has an antiproliferative effect in cancers, such as breast cancer, leukemia, and glioblastoma multiforme $(8,9,20)$. In the study by Shaimerdenova et al. (8), it was found that acyclovir decreased cell proliferation and increased apoptosis in the MCF7 breast cancer cell line. Due to this feature, it has been stated that acyclovir can be used in the treatment of breast cancer. In the study of Benedetti et al. (9) on leukemia cell lines, it was determined that acyclovir increased apoptosis in Jurkat (acute T-cell leukemia), U937, and K562 leukemia cell lines, and it was stated that it could be used together with chemotherapeutic drugs in the treatment. In the study of Kominsky et al. (20), it was revealed that the antiproliferative effect of acyclovir in glioblastoma multiforme cell lines is higher than in normal human astrocyte cell lines. Based on these studies, it was investigated whether acyclovir has an effect on angiogenesis in tumor cells. In the literature review, Lu et al. (21) found that acyclovir decreased the expression level of vascular endothelial growth factor and vascular endothelial growth factor receptor 2 in mouse renal cells. Even though there are studies investigating acyclovir's effects on cancer cell lines as discussed above, there has been a lack of studies conducted in current literature investigating the effect of acyclovir on angiogenesis $(22,23)$. From this point of view, we examined the effect of acyclovir on angiogenesis in the CAM model. In our study, acyclovir was applied at different concentrations. As a result of the experiment, toxic effects were observed in CAM models administered at $28.4 \mathrm{mg} / \mathrm{mL}$, while very weak anti-angiogenic effects were observed at a concentration of $3.55 \mathrm{mg} / \mathrm{mL}$. The crystallized membranes observed at the concentration of $7.1 \mathrm{mg} / \mathrm{mL}$ and $14.2 \mathrm{mg} /$ $\mathrm{mL}$ have been attributed to local crystallization reaction, which is also a well-known side effect of acyclovir, mentioned in literature as acyclovir induced crystal nephropathy (24). The local hyperosmotic reaction is a known disadvantage of the CAM model. It is known that certain substances in the form of salt crystals can cause hyperosmotic damage to the CAM membrane (25). It should be remembered that this reaction may occur in further CAM studies with acyclovir, and the assay should be planned accordingly.

The limitations of our study were that the study was conducted in an observational model and therefore its effect on angiogenesis was not examined with quantitative methods or at the gene level. However, a scoring system was used regarding the very weak anti-angiogenic effect of acyclovir (Table 1). Also, the anti-angiogenic effect needs to be confirmed by conducting studies on factors such as vascular endothelial growth factor gene level, or in vitro cell culture models as well as in vivo models (24).

We recommend that those who will study acyclovir on the CAM model in the future uses doses less than $7.1 \mathrm{mg} / \mathrm{mL}$ due to both the local non-specific reactions of the CAM model and the known crystallization reaction of acyclovir. In addition, increasing the number of eggs used in CAM experiments between $3.55 \mathrm{mg} /$ $\mathrm{mL}$ and $7.1 \mathrm{mg} / \mathrm{mL}$ acyclovir doses will facilitate the observational evaluation of the study. On a further note, the amount of the systemically administered dose of acyclovir reaching the area where the corresponding tissue is located will be responsible for the targeted antiangiogenic effect in cancer treatments. The amount of the administered dose does not reach this area completely. Therefore, the doses administered in this study are not necessarily the doses for the antiangiogenic effect in humans, they correspond to higher doses in this study. These doses should be assessed in microgram/ $\mathrm{mL}$ to further understand the therapeutic concentrations reaching the exact affected area for humans.

In conclusion, we observed very weak anti-angiogenic effects of acyclovir on the CAM model at a concentration level. It is known that anti-angiogenic agents can cause positive effects by disrupting tumor neovascularization (26). Some publications concluded that acyclovir has an antiproliferative effect on breast cancer, leukemia, and glioblastoma multiforme $(8,9,20)$. Extensive studies are needed on whether acyclovir can be used as adjuvant therapy in cancer treatment. Further studies are also needed to study the different potential effects of acyclovir on angiogenesis and perform quantitative analysis.

\section{Ethics Committee Approval: N/A}

Informed Consent: N/A

Conflict of Interest: The authors declare no conflict of interest.

Author Contributions: Concept: IK, BND, AE, ESA. Design: IK, BND, AE, ESA. Supervision: IK, BND, AE, ESA. Resources: IK, BND. Materials: BND, AE, ESA. Data collection and/or processing: BND, AE, ESA. Analysis and/ or Interpretation: IK, AE. Literature Search: IK, BND. Writing Manuscript: IK, BND. Critical Review: IK, BND, AE, ESA.

Financial Disclosure: The authors declared that this study received no financial support.

Acknowledgments: We would like to thank Taner Özgürtaş from the department of Medical Biochemistry of the University of Health Sciences Gülhane School of Medicine for their valuable guidance and contributions.

\section{REFERENCES}

1. Balfour HH. Antiviral drugs. N Engl J Med 1999;340(16):1255-68.

2. Gnann JW, Barton NH, Whitley RJ. Acyclovir: mechanism of action, pharmacokinetics, safety, and clinical applications. Pharmacotherapy 1983;3(5):275-83.

3. Razonable RR. Antiviral drugs for viruses other than human immunodeficiency virus. Mayo Clin Proc 2011;86(10):1009-26.

4. De Clercq E. Antiviral drugs in current clinical use. J Clin Virol 2004;30(2):11533.

5. De Clercq E, Li G. Approved antiviral drugs over the past 50 years. Clin Microbiol Rev 2016;29(3):695-747.

6. Gold D, Corey L. Acyclovir prophylaxis for herpes simplex virus infection. Antimicrob Agents Chemother 1987;31(3):361-7. 
7. Field HJ, Goldthorpe SE. Antiviral drug resistance. Trends Pharmacol Sci 1989;10(8):333-7.

8. Shaimerdenova M, Karapina O, Mektepbayeva D et al. The effects of antiviral treatment on breast cancer cell line. Infect Agents Cancer 2017;12(1):18.

9. Benedetti S, Catalani S, Palma F et al. Acyclovir induces cell cycle perturbation and apoptosis in jurkat leukemia cells and enhances chemotherapeutic drug cytotoxicity. Life Sci 2018;215:80-5.

10. Lokman NA, Elder ASF, Ricciardelli C et al. Chick chorioallantoic membrane (CAM) assay as an in vivo model to study the effect of newly identified molecules on ovarian cancer invasion and metastasis. Int J Mol Sci 2012;13(8):9959-70.

11. Nowak-Sliwinska P, Segura T, Iruela-Arispe ML. The chicken chorioallantoic membrane model in biology, medicine, and bioengineering. Angiogenesis 2014;17(4):779-804

12. FDA-approved drugs (website) (cited 2021 October 10). Available from: URL: https://www.accessdata.fda.gov/scripts/cder/daf/index.cfm?event=overview.process\&ApplNo $=020089$.

13. Zhou Q, Qi C, Li Y et al. A novel four-step system for screening angiogenesis inhibitors. Mol Med Rep 2013;8:1734-40.

14. Bürgermeister J, Paper DH, Vogl H et al. LaPSvSd1, a (1-->3)-beta-galactan sulfate and its effect on angiogenesis in vivo and in vitro. Carbohydr Res 2002;337(16):1459-66.

15. Paper DH, Vogl H, Franz G. Low-molecular-weight galactan sulfates as angiogenesis inhibitors. Contribu to Oncol 1999;54:191-9.

16. Karaman H, Tufek A, Karaman E et al. Opioids inhibit angiogenesis in a chorioallantoic membrane model. Pain Phys 2017;20(2):11-21.
17. Kilıçcalan İ, Erkovan A, Sen E. Caenorhabditis elegans and angiogenesis. Turkish Med Stud J 2019;6(1):37-41.

18. Brand $\mathrm{M}$, Lamandé N, Larger E et al. Angiotensinogen impairs angiogenesis in the chick chorioallantoic membrane. J Mol Med 2007;85(5):451-60.

19. Ribatti D. The chick embryo chorioallantoic membrane (CAM). A multifaceted experimental model. Mech Dev 2016;141:70-7.

20. Kominsky SL, Subramaniam PS, Johnson HM et al. Inhibitory effects of IFN-gamma and acyclovir on the glioblastoma cell cycle. J Interferon Cytokine Res 2000;20(5):463-9.

21. Lu H, Han Y-J, Xu J-D et al. Proteomic characterization of acyclovir-induced nephrotoxicity in a mouse model. PLoS One (serial online) 2014;9(7). Available from: URL: https://journals.plos.org/plosone/article?id=10.1371/journal. pone. 0103185

22. Rajabi M, Mousa SA. The role of angiogenesis in cancer treatment. Biomedicines 2017;5(2):34.

23. Lugano R, Ramachandran M, Dimberg A. Tumor angiogenesis: causes, consequences, challenges and opportunities. Cell Mol Life Sci 2019;77(9):1745-70.

24. Fleischer R, Johnson M. Acyclovir nephrotoxicity: a case report highlighting the importance of prevention, detection, and treatment of acyclovir-induced nephropathy. Case Rep Med (serial online)2010. Available from: URL: https://www. ncbi.nlm.nih.gov/pmc/articles/PMC2939444/.

25. West DC, Thompson WD, Sells PG et al. Angiogenesis assays using chick chorioallantoic membrane. Methods Mol Med 2001;46:107-29.

26. Hillen F, Griffioen AW. Tumour vascularization: sprouting angiogenesis and beyond. Canc Metastasis Rev 2007;26(3-4):489-502. 